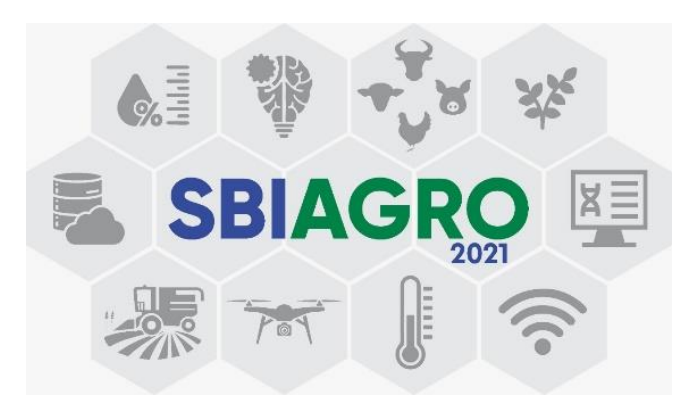

\title{
Impacto das mudanças climáticas no período 2040-2080 sobre a aptidão do cultivo de Coffea arabica no Brasil
}

\author{
João Antonio Lorençone ${ }^{1}$, Lucas E. de O. Aparecido ${ }^{2}$, Pedro Antonio Lorençone ${ }^{1}$, \\ Guilherme Botega Torsoni ${ }^{1}$ \\ ${ }^{1}$ Instituto Federal de Mato Grosso do Sul (IFMS) \\ Naviraí - MS - Brazil \\ ${ }^{2}$ Instituto Federal do Sul de Minas (IFSULDEMINAS) \\ Muzambinho - MG - Brazil \\ joao.lorencone@estudante.ifms.edu.br, \\ lucas.aparecido@muz. ifsuldeminas.edu.br, \\ pedro.lorenconedestudante.ifms.edu.br, \\ guilherme.torsoni.ifms.edu.br
}

\begin{abstract}
The objective of this work was to evaluate the impact of climate change on areas suitable for Arabica coffee cultivation under different climate change scenarios. The work was carried out for all of Brazil, using data from 1960-2020. The BCC - CSM 1 climate model was used to obtain future climate data for all RCP 2.6, 4.5, 6.0, 8.5 scenarios. All future scenarios of climate change showed a reduction in the total areas suitable for coffee cultivation in Brazil, with an average reduction of 50\%. Furthermore, areas restricted by excessive heat and water deficiency were the most common throughout the country in the future scenarios, with an average of $63 \%$ of the entire territory.
\end{abstract}

Resumo. O objetivo desse trabalho foi avaliar o impacto das mudanças climáticas sobre as áreas aptas para o cultivo do café arábica nos diferentes cenários de mudanças climáticas. O trabalho foi realizado para todo o Brasil, sendo utilizado dados no período de 1960-2020. Ainda, para obtenção dos dados climáticos futuros foi utilizado o modelo climático o BCC-CSM 1, de todos os cenários RCP 2.6, 4.5, 6.0, 8.5. Todos os cenários futuros de mudanças climáticas demonstraram redução no total de áreas aptas ao cultivo do cafeeiro no Brasil, com redução média de 50\%. Ainda, as áreas com restrição por excesso térmico e deficiência hídrica foram as mais comuns por todo o país nos cenários futuros, com média de 63\% de todo território.

\section{Introdução}

O cultivo de café tem papel fundamental na geração de empregos e renda, estando muito presente na agricultura familiar (Muñoz-Rios et al. 2020). É uma das commodities mais 
comercializadas no mundo, sendo o Brasil o maior produtor e exportador do grão (WTO 2020).

As mudanças climáticas se tornaram um grande problema no século 21. Estudos comprovam que a produção de alimento será impactada negativamente, ao mesmo tempo em que a população continua com seu crescimento. No último século, a temperatura global aumentou $1{ }^{\circ} \mathrm{C}( \pm 0,2)$, as previsões para 2100 são de aumentos entre $1,5^{\circ} \mathrm{C}$ e 6 ${ }^{\circ} \mathrm{C}$ (IPCC, 2018). Essas condições podem representar um risco para a vegetação devido ao aumento da evaporação, períodos mais longos de seca e redução disponibilidade de água no solo para as plantas.

Dessa forma, o objetivo desse estudo é avaliar o impacto das mudanças climáticas sobre as áreas aptas para o cultivo do café arábica nos diferentes cenários de mudanças climáticas.

\section{Material e Métodos}

Foram coletados os elementos climáticos Temperatura do ar $\left({ }^{\circ} \mathrm{C}\right)$ e Precipitação $(\mathrm{mm})$, de 4.947 estações meteorológicas do Banco de Dados Meteorológicos para Ensino e Pesquisa (BDMEP) do Instituto Nacional de Meteorologia do Brasil - INMET (Brasil, 1992), no período de 1960-2020. Os dados provenientes do IPCC são resultados de simulações de modelos globais de alguns centros de pesquisa que contribuem para a confecção do relatório do IPCC-AR5, forçadas pelas concentrações observadas de gases de efeito estufa durante o século XX e forçadas por uma concentração estimada para $\mathrm{o}$ século XXI (IPCC, 2013). O modelo climático global utilizado foi o BCC - CSM 1.1 desenvolvido no Centro Climático de Pequim (BCC), possui resolução de 125 x $125 \mathrm{~km}$, com 26 níveis verticais, seus componentes são: atmosfera, superfície de terra, oceano, gelo marinho, ciclo do carbono terrestre, ciclos biogeoquímicos do oceano (Flato et al., 2014). Os dados climáticos dos cenários RCPs foram coletados através da plataforma WorldClim 2.

Para poder identificar as áreas aptas, restritas ou inaptas ao cultivo do café foram utilizadas as faixas de aptidão climáticas ideais de temperatura do $\operatorname{ar}\left({ }^{\circ} \mathrm{C}\right)$, temperatura do ar do mês de novembro $\left({ }^{\circ} \mathrm{C}\right)$, temperatura do ar do mês mais frio do ano $\left({ }^{\circ} \mathrm{C}\right)$ e déficit hídrico para o C.arabica, conforme os seguintes autores Camargo (1977), Matiello (1991) e Camargo and Pereira (1994). Um fluxograma explicativo das etapas de elaboração do trabalho pode ser acessado a partir do link:

https://photos.app.goo.gl/sgXYvLXeJP75vJ4g6

Os softwares utilizados no trabalho foram o Quantum GIS (QGis) 3.16.4 para interpolação dos dados e elaboração do mapeamento, Python 3.10.0 e o Excel Versão 2109 para elaboração das análises estatísticas.

\section{Resultados e Discussão}

O zoneamento agroclimático do Coffea arabica nos cenários de mudanças climáticas, apresentou um aumento significativo das áreas inaptas, com grande redução das áreas aptas ao cultivo do cafeeiro (Figura 1 E). Grande parte do estado de Minas Gerais, apresentou redução das áreas aptas em todos os cenários, da mesma maneira para o estado de São Paulo. Por outro lado parte da região sul do país, passaram de inaptas por insuficiência térmica para aptas ao cultivo (Figura 1). Essas alterações de aptidões 
climáticas no Brasil podem resultar em redução da produção de café, tendo em vista que as regiões com maiores altitudes no país podem apresentar restrições climáticas e grande parte das possíveis novas regiões aptas ao cultivo não possuem uma altitude que estimule o melhor desenvolvimento do cafeeiro (Carvalho et al., 2019).

Grande parte do território brasileiro demostrou mudar de classe nos cenários possíveis de mudanças climáticas em relação ao atual, tanto nos cenários de 2041 - 2060 quanto nos de 2061 - 2080 (Figura 1). Regiões como o oeste de Mato Grosso e o estado do Mato Grosso do Sul passaram das classes com restrição por excesso térmico para restrição térmica e hídrica, demostrando que o possível aumento do déficit hídrico resultará em grandes impactos às lavouras de café, tendo em vista que o café é muito suscetível ao estresse hídrico. A utilização de irrigação complementar para os cafeeiros pode ser uma alternativa para contornar as possíveis cenários e mudanças climáticas, proporcionando um impacto menor na produção final das lavouras do país (Moreira et al. 2021).
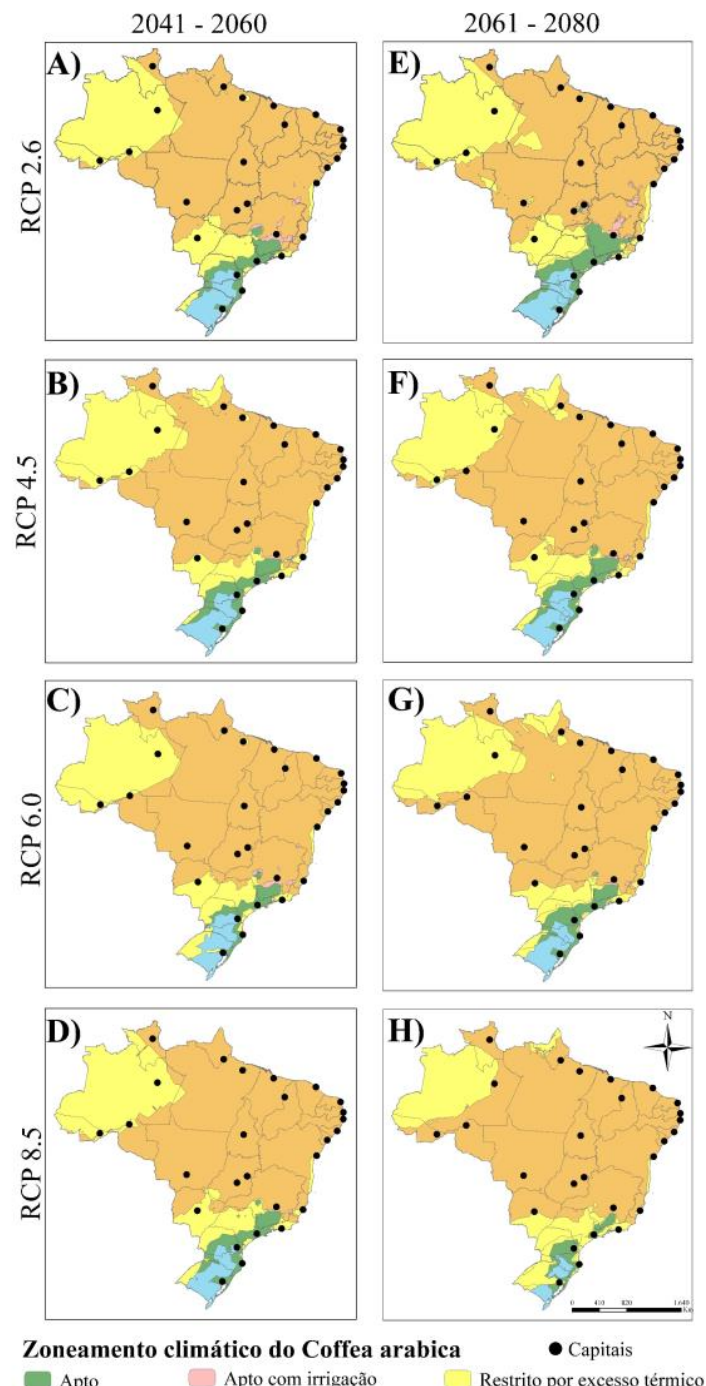

Zoneamento climático do Coffea
Apto $\square$ Apto com irrigação Restrito por excesso térmico

Restrito por excesso térmico e deficiência hídrica $\square$ Restrito por insuficiência térmica

Figure 1. Zoneamento climático do Coffea arábica para o Brasil nos diferentes cenários de mudanças climática. 


\section{Conclusões}

As mudanças climáticas futuras podem impactar negativamente a cultura do cafeeiro em todos os cenários RCPs estudados. Todos os cenários do modelo BCC - CSM 1.1 apresentam redução no total de áreas aptas ao cultivo do cafeeiro no Brasil, com redução média de 50\%. Ainda, as áreas com restrição por excesso térmico e deficiência hídrica são as mais comuns por todo o país nos cenários futuros, com média de $62,95 \%$ de todo território.

\section{References}

Camargo, A. de. (1977). Zoneamento de aptidão climática para a cafeicultura de arábica e robusta no Brasil. Fundação IBGE, Recursos, meio ambiente e polvição, 68-76.

Camargo, A. de, \& Pereira, A. (1994). Agrometeorology of the coffee crop. Geneva: World Meteorological Organization, 92.

Carvalho, C. F., Carvalho, S. M., \& Souza, B. (2019). Coffee. In B. Souza, L. L. Vázquez, \& R. C. Marucci (Orgs.), Natural Enemies of Insect Pests in Neotropical Agroecosystems (p. 277-291). Springer International Publishing. https://doi.org/10.1007/978-3-030-24733-1_23

Flato, G., Marotzke, J., Abiodun, B., Braconnot, P., Chou, S. C., Collins, W., Cox, P., Driouech, F., Emori, S., \& Eyring, V. (2014). Evaluation of climate models. In Climate change 2013: The physical science basis. Contribution of Working Group I to the Fifth Assessment Report of the Intergovernmental Panel on Climate Change (p. 741-866). Cambridge University Press.

IPCC. (2018). Global Warming of $1.5^{\circ} \mathrm{C}$, Summary for Policymakers. Contribution of Working Groups I, II and III to the Fifth Assessment Report of the Intergovernmental Panel on Climate Change. INTERGOVERNMENTAL PANEL ON CLIMATE CHANGE.

Matiello, J. B. (1991). O café: Do cultivo ao consumo. Editora Globo São Paulo.

Moreira, T. R., da Silva, S. F., da Silva, N. B., dos Santos, G. M. A. D. A., \& dos Santos, A. R. (2021). Global Warming and the Effects of Climate Change on Coffee Production. In L. Louzada Pereira \& T. Rizzo Moreira (Orgs.), Quality Determinants In Coffee Production (p. 65-100). Springer International Publishing. https://doi.org/10.1007/978-3-030-54437-9_2

Muñoz-Rios, L. A., Vargas-Villegas, J., \& Suarez, A. (2020). Local perceptions about rural abandonment drivers in the Colombian coffee region: Insights from the city of Manizales. Land Use Policy, 91, 104361. https://doi.org/10.1016/j.landusepol.2019.104361

WTO, W. T. O. (2020). Statistics on merchandise trade. WORLD TRADE ORGANIZATION. timeseries.wto.org/ 